\title{
Controlling the quantum rotational dynamics of a driven planar rotor by rebuilding barriers in the classical phase space ${ }^{\dagger}$
}

\author{
ARCHANA SHUKLA and SRIHARI KESHAVAMURTHY* \\ Department of Chemistry, Indian Institute of Technology, Kanpur, Uttar Pradesh 208 016, India \\ E-mail: srihari@iitk.ac.in
}

MS received 3 February 2017; accepted 10 April 2017

\begin{abstract}
The present work aims to control the rotational excitations of an ac-driven planar rotor, a model for rigid diatomic molecules, by rebuilding barriers in the classical phase space. The barriers are invariant tori with irrational winding ratios which are perturbatively constructed at desired locations in the phase space. We establish that constructing such barriers, equivalent to additional weak fields, can efficiently suppress the chaos leading to the control of various processes. The phase space barriers are shown to be effective in controlling the quantum dynamics as well. In particular, the efficiency of the phase space barriers towards controlling dynamical tunneling in the system is explored. Our studies are relevant to understanding the role of the chaotic regions in dynamical tunneling and for molecular alignment using bichromatic fields.
\end{abstract}

Keywords. Rigid rotor; dynamical tunneling; coherent control; phase space barriers; molecular alignment.

\section{Introduction}

Starting with the early proposals ${ }^{1-3}$ for coherently controlling quantum phenomena using tailored fields there has been an increasing interest ${ }^{4-7}$ to uncover the control mechanism as encoded in the structure of the applied fields. Following the initial studies there was a general consensus that coherent control utilizes ${ }^{8}$ the quantum interference effect between different pathways that connect a given initial and final state. In this sense coherent control can be viewed as an intrinsically quantum technique with no classical analog. Recently, however, doubts have been cast on this long held viewpoint. In particular, it is now understood that a typical laser control scenario, involving ac-fields with two different frequency components, is not equivalent to the Young's double slit paradigm. ${ }^{9}$ Surprisingly, in the so called laser-induced symmetry breaking approach the control mechanism has a common origin from both classical and quantum perspectives. ${ }^{10,11}$ The reasons behind this unusual viewpoint has to do with the fact that the nonlinear response of a system to the combined fields has a well defined classical limit. ${ }^{10,12}$ Indeed, it has been argued that the symmetry requirements on the field to generate phase-controllable dynamics are identical ${ }^{13}$ in both classical and quantum mechanics.

\footnotetext{
*For correspondence

${ }^{\dagger}$ Dedicated to the memory of late Professor Charusita Chakravarty.
}

To a large extent the renewed understanding of coherent control mechanisms mentioned above has come about due to a sustained interest in the classical-quantum correspondence studies of driven systems. Such studies in atomic, molecular and mesoscopic systems have established the influence of the classical phase space on a wide range of quantum phenomena. The literature in this context is substantial and as examples of early work we mention, microwave ionization of atoms, ${ }^{14}$ dissociation of diatomic molecules using continuous ${ }^{15}$ and chirped fields, ${ }^{16}$ dynamical tunneling, ${ }^{17}$ and even quantum entanglement. ${ }^{18}$ Every one of these phenomena exhibit sensitive dependence on various classical phase space structures including chaos, cantori, and nonlinear resonances. A partial list of reviews, ${ }^{19-29}$ spanning a wide range of disciplines, gives a glimpse of the utility of the classical-quantum correspondence viewpoint.

Thus, given that laser-induced symmetry breaking has a classical origin and that driven systems are cognizant of the nature of the classical phase space, is it feasible to exert control by suitable modification of the classical phase space structures? By suitable modification we mean reducing the extent of chaos or perturbing a specific nonlinear resonance by introducing additional, possibly weak, control terms to the original classical Hamiltonian. Starting with the initial work ${ }^{30}$ by Ciraolo et al., there are now several examples for such a local phase space based approach to controlling classical dynamics. ${ }^{31-35}$ However, it is crucial to determine the 
efficiency of the phase space barriers in the corresponding quantum system since there can be a subtle interplay between classical and quantum transports depending on the effective Planck constant of the system. In this regard there has not been much work except for an earlier study on the influence of the rebuilt local phase space barriers on the quantum dynamics of a ac-driven Morse oscillator. ${ }^{36}$ Moreover, as seen in the earlier work ${ }^{36}$ and the current work, it is advantageous if one can map the control terms to additional fields which, apart from being experimentally accessible, can yield valuable insights into the mechanisms of laser-induced symmetry breaking.

In the present study we take a fairly simple, yet paradigmatic, model of a driven planar rotor and show that it is possible to control both the quantum and classical rotational dynamics by modifying the classical phase space. In Sec. 2 we provide a detailed introduction to the model, nature of the phase space, and the motivations for our interest in the specific system. In Sec. 3 we give a brief but essential discussion of the ideas and techniques for the local phase space control and interpret the control terms. This is followed by results where in Sec. 4.1 we demonstrate the efficiency of controlling the quantum rotational excitations and in Sec. 4.2 establish that it is possible to control the dynamical tunneling process as well. Finally, in Sec. 5 we summarize the present work and briefly mention aspects of the control method that needs further attention.

\section{Model Hamiltonian}

The Hamiltonian of interest ${ }^{37}$ is a periodically driven planar rotor given by

$$
H(J, \theta, t)=\frac{J^{2}}{2 I}-\mu \mathcal{E}_{0} \cos \omega t \cos \theta
$$

where the external ac-driving field with amplitude $\mathcal{E}_{0}$ and frequency $\omega$ makes an angle $\theta$ with the dipole moment vector of magnitude $\mu$. The moment of inertia of the rotor is denoted by $I$. The Hamiltonian can be expressed as

$$
\begin{aligned}
H(J, \theta, t) & =\frac{J^{2}}{2 I}-\frac{f}{2}[\cos (\theta+\omega t)+\cos (\theta-\omega t)] \\
& \equiv H_{0}(J)-\epsilon[\cos (\theta+\omega t)+\cos (\theta-\omega t)]
\end{aligned}
$$

with $f \equiv 2 \epsilon=\mu \mathcal{E}_{0}$. Note that the Hamiltonian above arises in a number of different contexts. For instance, it is possible to interpret Eq. 2 as the Hamiltonian, in action-angle representation, for a ac-driven pendulum with only two resonance terms being retained. ${ }^{38}$ As a further example, Eq. 2 arises in experiments ${ }^{39,40}$ on cold atoms trapped in a time modulated optical lattice. Following earlier work, ${ }^{37}$ we work with the scaled Hamiltonian

$$
H(J, \theta, t)=\frac{1}{2} J^{2}-\epsilon[\cos (\theta+\omega t)+\cos (\theta-\omega t)]
$$

with $\omega=1$. In the above Hamiltonian the various parameters are dimensionless. The rescaling results in an effective Planck constant $\hbar_{\text {eff }}$, henceforth denoted as $\hbar$, in the context of the quantum studies.

The phase space of the system for Eq. 3 is shown in Figure 1 as a stroboscopic surface of section for two values of the field strength. The two prominent islands of regularity at $J_{ \pm}^{r} \approx \pm 1$ are the two 1:1 nonlinear resonances arising from the field-matter interaction. These hindered rotational motion (librations) are determined by the resonance condition

$$
\left.\frac{\partial H_{0}(J)}{\partial J}\right|_{J=J_{ \pm}^{2}}=\Omega\left(J_{ \pm}^{r}\right)= \pm \omega
$$

with $\Omega$ being the nonlinear frequency of the rotor. A qualitative understanding of the nature of the phase space can be obtained using Chirikov's resonance overlap theory. ${ }^{41}$ For small values of the field strength $f$ the two resonances do not overlap and each has a full width of $\Delta J_{\max }=\sqrt{8 f}$. With increasing field strength the resonances overlap at $f=$

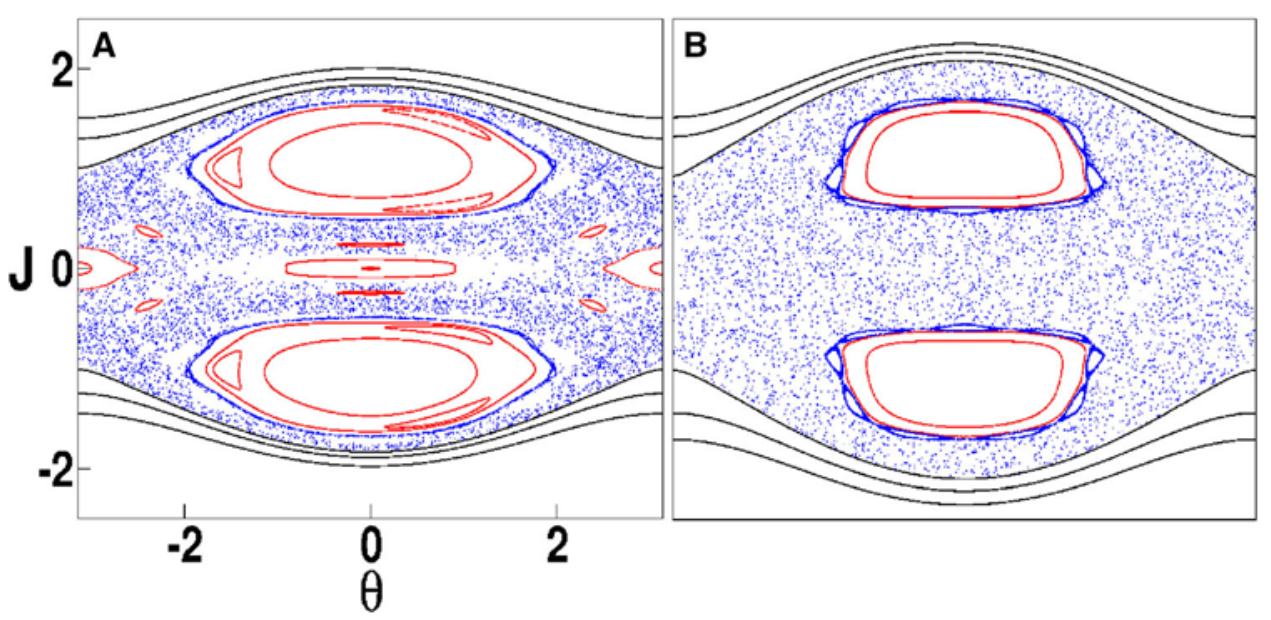

Figure 1. Stroboscopic surface of section for the Hamiltonian Eq. 3 for (A) $f=0.3$ and (B) $f=0.5$ with driving field frequency $\omega=1$. 
$f_{c}=1 / 2$ and for $f>f_{c}$ generate substantial chaos in the phase space. Semiclassically, the width of a resonance translates to the number of quantum states that are trapped in the resonance zones. We refer the reader to the earlier work by Berman, Zaslavsky, and Kolovsky ${ }^{42-44}$ for further details on the classical-quantum correspondence for transition to stochasticity in such double resonance models.

The Hamiltonian of interest affords several advantages in terms of a detailed classical-quantum correspondence study of various phenomena. Since the system possess the dynamical symmetry $H(-J,-\theta, t)=H(J, \theta, t)$, the classical phase space exhibits symmetry related structures and one expects dynamical tunneling ${ }^{17}$ between such structures. Thus, classical trajectories initiated in the upper 1:1 island in Figure 1 are trapped forever whereas quantum dynamics allows an initial quantum state localized in the upper island to dynamically tunnel to the symmetry related lower $1: 1$ island. The simple field-matter resonance structure of Eq. 3 allows for a clear analysis of dynamical tunneling effects in terms of the various nonlinear resonances ${ }^{45,46}$ and chaos, ${ }^{47}$ as shown in an earlier study ${ }^{48}$ by Mouchet, Eltschka, and Schlagheck. Moreover, an important point to note from Figure 1 is that the chaotic region is bounded from above and below by KAM tori corresponding to free rotation. This is in contrast to examples such as the kicked rotor, ${ }^{49,50}$ microwave driven atoms ${ }^{51,52}$ and driven Morse oscillator ${ }^{15}$ wherein the chaotic regions are unbounded and there can be strong quantum localization effects in the classically chaotic region. Indeed, detailed studies by Moiseyev, Korsch, and Mirbach have shown ${ }^{37}$ that in case of the periodically driven rotor the quasienergy states are extended and do not exhibit strong quantum localization effects. Consequently, even when the field is far off-resonance from the typical rotational frequencies, an initial rotational quantum state (for example $j=0$ with $f=0.5$ as in Figure 1B) can transfer population to highly excited rotational states with almost equal probabilities - the maximal excited rotational state quantum number being determined by the extent of the bounded chaotic region in the classical phase space. ${ }^{37}$

Given the close classical-quantum correspondence for the driven rotor one expects that it should be possible to control quantum processes like dynamical tunneling and rotational excitation by suitable modifications of the classical phase space. In particular, since the chaos in the classical phase space plays an important role, it is of interest to study the effect of "bottling up" of chaos on the quantum processes of interest. One way to implement this idea is to locally modify the phase space by rebuilding the KAM barriers in the phase space to effectively confine and/or reduce the extent of stochasticity. In the following section we outline such an approach due to Vittot and coworkers ${ }^{31,32}$ and derive the explicit form of the control terms for the driven rotor system.

\section{Local phase space control}

We start by briefly recounting the local phase control idea as described ${ }^{33}$ in Huang et al., and follow their notation, as far as possible, for convenience and clarity. The key features of the method are summarized in a general setting followed by implementation for our specific model Hamiltonian.

Consider the general Hamiltonian of the form

$$
H(\mathbf{A}, \boldsymbol{\phi})=H_{0}(\mathbf{A})+\epsilon V(\mathbf{A}, \boldsymbol{\phi})
$$

with $(\mathbf{A}, \boldsymbol{\phi}) \in \mathbb{R}^{f} \times \mathbb{T}^{f}$ being the $f$-dimensional action-angle variables. For $\epsilon=0$ the phase space is integrable and foliated by invariant tori with frequency $\boldsymbol{\Omega}(\mathbf{A})=\partial H_{0}(\mathbf{A}) / \partial \mathbf{A}$. For increasing values of $\epsilon$ an increasing number of the invariant tori break up as in the classic KAM scenario for nonlinear, and nondegenerate, Hamiltonians. In particular, resonant tori satisfying $\mathbf{k} \cdot \boldsymbol{\Omega}=0$ for nonzero integer vector $\mathbf{k}$ are the first ones to be destroyed by the perturbation whereas non-resonant tori are destroyed beyond a threshold value $\epsilon_{c}$ of the perturbation strength. The goal of the local phase space control is to rebuild a specific non-resonant invariant torus for $\epsilon>\epsilon_{c}$ by modifying the original Hamiltonian in Eq. 5 as

$$
H_{c}(\mathbf{A}, \boldsymbol{\phi})=H_{0}(\mathbf{A})+\epsilon V(\mathbf{A}, \boldsymbol{\phi})+h(\boldsymbol{\phi})
$$

The control term is $O\left(\epsilon^{2}\right)$ i.e., small with respect to the perturbation $\epsilon V(\mathbf{A}, \boldsymbol{\phi})$ in the original uncontrolled Hamiltonian. Assuming that the non-resonant torus with frequency $\boldsymbol{\Omega}$ is located at $\mathbf{A}=\mathbf{A}_{0}$, the expression for the control term can be written as

$$
h(\boldsymbol{\phi})=-H\left(\mathbf{A}_{0}-\partial_{\boldsymbol{\phi}} \Gamma b(\boldsymbol{\phi}), \boldsymbol{\phi}\right)
$$

where $b(\boldsymbol{\phi}) \equiv H\left(\mathbf{A}_{0}, \boldsymbol{\phi}\right)=\sum_{\mathbf{k} \in \mathbb{Z}^{f}} b_{\mathbf{k}} e^{i \mathbf{k} \cdot \boldsymbol{\phi}}$, and $\Gamma$ is a linear operator defined by

$$
\Gamma b(\boldsymbol{\phi})=\sum_{\mathbf{k} \cdot \boldsymbol{\Omega} \neq 0} \frac{b_{\mathbf{k}}}{i \mathbf{k} \cdot \boldsymbol{\Omega}} e^{i \mathbf{k} \cdot \boldsymbol{\phi}}
$$

Note that the condition $\mathbf{k} \cdot \boldsymbol{\Omega} \neq 0$ in the equation above is due to the fact that $\boldsymbol{\Omega}$ is chosen to be non-resonant. In addition, the rebuilt invariant torus for the controlled system is located at

$$
\mathbf{A}=\mathbf{A}_{0}-\partial_{\phi} \Gamma b(\boldsymbol{\phi})
$$

Details regarding the regimes of validity of the local control strategy as well as an explicit construction of the control term via suitably chosen canonical transformations can be found in the work ${ }^{32}$ of Vittot et al. In this work we apply the above equations to the Hamiltonian of interest and numerically test the effectiveness of the control term.

\subsection{Control term for the driven rotor case}

In order to apply the local phase space control approach to the Hamiltonian in Eq. 3, we start by converting the 
time-dependent system to an autonomous system. So, we consider $t$ as an additional angle variable whose conjugate action is the energy $E$. The resulting autonomous Hamiltonian can be expressed as

$$
\mathcal{H}(\mathbf{A}, \boldsymbol{\phi})=E+\frac{1}{2} J^{2}-\epsilon[\cos (\theta+t)+\cos (\theta-t)]
$$

where the action and angle variables are denoted by $\mathbf{A} \equiv(J, E)$ and $\boldsymbol{\phi} \equiv(\theta, t)$ and $\boldsymbol{\Omega}=(\Omega, 1)$ being the frequency vector.

Since our purpose is to rebuild irrational barriers in the phase space we choose the ratio of the field to rotor frequencies as

$$
\frac{\omega}{\Omega}=g+\gamma
$$

with $g$ being an integer and $\gamma=(\sqrt{5}-1) / 2 \approx 0.618$ is the golden ratio. Working with the scaled Hamiltonian, our tori to be rebuilt are located at $\mathbf{A}_{0}=\left(J_{g}, 0\right)$ with frequency $\boldsymbol{\Omega}=\left(\Omega_{g}, 1\right)$ and $\Omega_{g}=J_{g}=(g+\gamma)^{-1}$. Note that the chosen frequency ratio corresponds to the so called noble frequencies, in general expressible as $(a \gamma+b) /(c \gamma+d)$ with $a d-b c= \pm 1$, which form ${ }^{53,54}$ fairly robust barriers for transport in the phase space of systems with two degrees of freedom. The case of Eq. 11 corresponds to $a=d=1, b=g$, and $c=0$.

Following the methodology discussed above the desired control term as given in Eq. 7 is

$$
h(\boldsymbol{\phi})=-\mathcal{H}\left(\mathbf{A}_{0}-\partial_{\boldsymbol{\phi}} \Gamma b(\boldsymbol{\phi}), \boldsymbol{\phi}\right)
$$

An explicit computation of the control term yields

$$
\begin{aligned}
h(\boldsymbol{\phi}) & =-\frac{1}{2}\left[\partial_{\theta} \Gamma b(\boldsymbol{\phi})\right]^{2} \\
& =-\frac{\epsilon^{2}}{2}\left[\frac{\cos (\theta+t)}{\Omega_{g}+1}+\frac{\cos (\theta-t)}{\Omega_{g}-1}\right]^{2} \\
& =-\frac{\epsilon^{2}}{4}\left[\frac{\cos (2 \theta+2 t)}{\left(\Omega_{g}+1\right)^{2}}+\frac{\cos (2 \theta-2 t)}{\left(\Omega_{g}-1\right)^{2}}+\frac{2 \cos 2 \theta}{\Omega_{g}^{2}-1}\right]
\end{aligned}
$$

where in the last line above terms independent of $\theta$ have been dropped since they are uninteresting from the dynamical perspective.

\subsection{Efficiency and an interpretation of the control term}

As expected, the control term in Eq. 13 is $O\left(\epsilon^{2}\right)$ and has three Fourier components $(2,2),(2,-2)$, and $(2,0)$. Interestingly, the modes $(2, \pm 2)$ can be interpreted as due to a second weak field with frequency $2 \omega$ at an angle $2 \theta$ with respect to the dipole vector. On the other hand, the $(2,0)$ mode corresponds to an additional static field with a relative phase shift of $\pi$ since $\left|\Omega_{g}\right|<1$. In other words, the control Hamiltonian can be thought of as equivalent to a Hamiltonian such as

$$
\begin{aligned}
K(J, \theta, t)= & H(J, \theta, t)-f_{2} \cos 2 t \cos 2 \theta \\
& -f_{s} \cos (2 \theta+\pi)
\end{aligned}
$$

with $f_{2}$ and $f_{s}$ being related to the $2 \omega$-field strength and static field strength respectively. Comparing to our explicit control Hamiltonian result in Eq. 13 one obtains

$$
\begin{aligned}
& \frac{f_{2}}{f} \equiv \frac{\epsilon}{4\left(\Omega_{g}-1\right)^{2}} \\
& \frac{f_{s}}{f} \equiv \frac{\epsilon}{4\left(1-\Omega_{g}^{2}\right)}
\end{aligned}
$$

Clearly, $f>f_{2} \geq f_{s}$ with the equality holding for large $g$ values i.e., nearly symmetric location of the rebuilt barrier. Consequently, both the $(2,-2)$ and the $(2,0)$ modes are expected to be crucial and, given the choice of our rebuilt torus action, the $(2,2)$ mode plays a relatively minor role.

The efficiency of the control term is illustrated in Figure 2 where the phase space of the controlled Hamiltonian with $\omega / \Omega=3+\gamma$ and $1+\gamma$ are shown for $f=0.3$ (A and B respectively) and $f=0.5$ (C and $\mathrm{D}$ respectively) are shown. On comparing to the uncontrolled phase spaces shown in Figure 1 it is clear that the addition of the control term has indeed constrained the stochasticity in the phase space to specific regions. In addition, with increasing $g$ in Eq. 11 the locations of the rebuilt tori move closer to $J \approx 0$. However, despite the nearly symmetric nature of the phase space for large $g$, the local phase space structure in the vicinity of the 1:1 islands has important differences in terms of the secondary resonances. In Figure 3 we show the influence of the specific Fourier modes in the control term. The result of neglecting the $(2,2)$ mode is shown in Figure 3B and indicates that the influence of the specific mode is minimal. Figure 3C shows that, despite the barriers being rebuilt, neglecting the $(2,-2)$ mode leads to some subtle changes in the phase space. For instance, the extent of chaos around the upper (lower) 1:1 island in Figure $3 \mathrm{C}$ is increased (decreased) in comparison to Figure $3 \mathrm{~A}$ and B. However, Figure 3D clearly shows that the $(2,0)$ mode i.e., the static field is crucial in order to rebuild the barrier. 

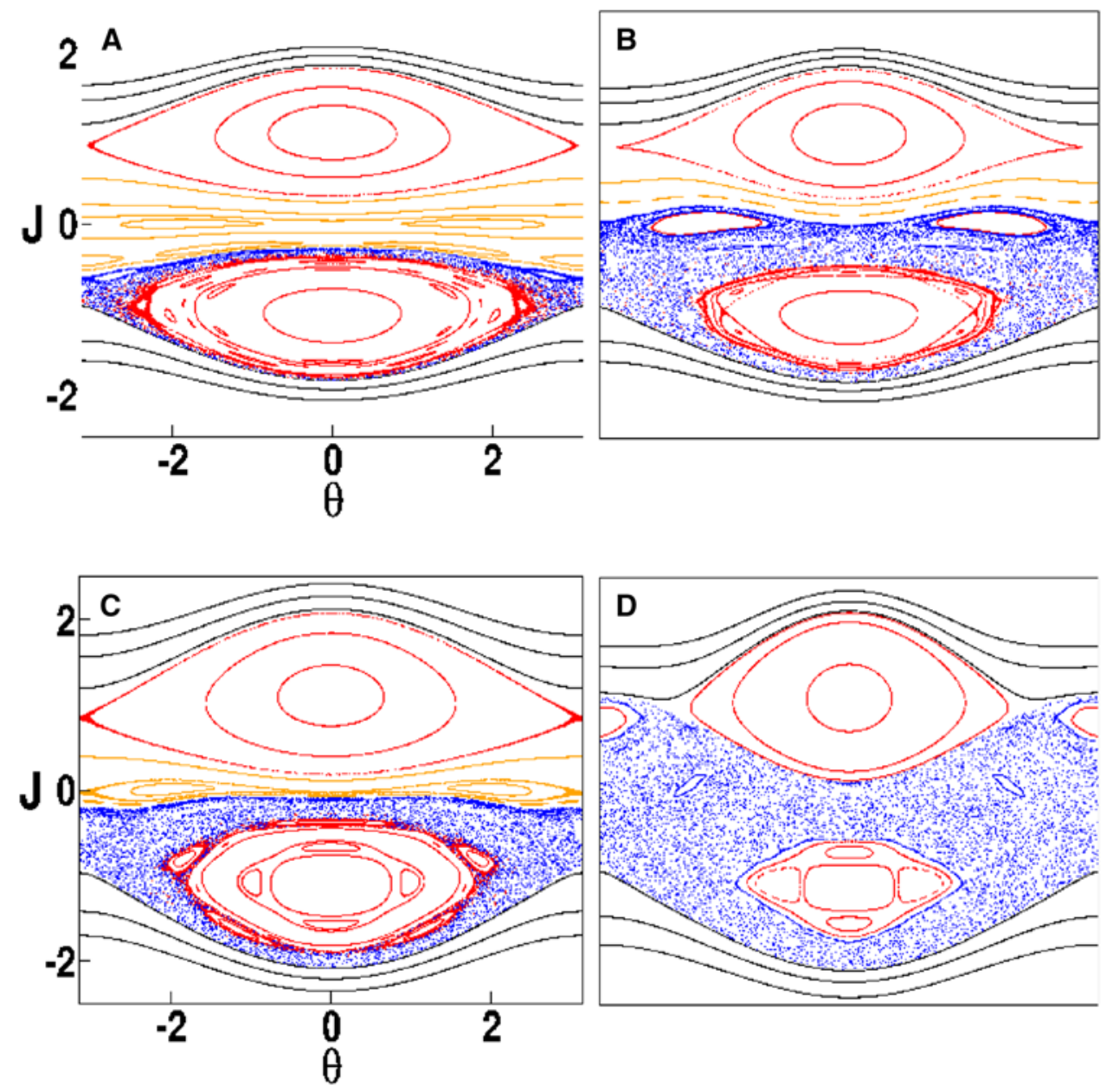

Figure 2. Stroboscopic surfaces of section showing the influence of the control field in Eq. 13 for two examples of the rebuilt KAM tori. The frequency ratio $\omega / \Omega$ equal to (A) $3+\gamma$ and (B) $1+\gamma$ for $f=2 \epsilon=0.3$. (C) and (D) show the corresponding phase spaces for $f=2 \epsilon=0.5$. All the plots have identical axes range.

\section{Results and Discussion}

We now investigate the extent to which the rebuilt classical phase space barriers influence specific quantum processes. In addition, we are interested in the classical-quantum correspondence of the control process. Therefore, we analyze the effect of the rebuilt phase space barriers on an exquisitely quantum phenomenon of dynamical tunneling between the resonant islands. In particular we analyze the example case considered in the earlier work ${ }^{48}$ by Mouchet, Eltschka, and Schlagheck wherein both resonance and chaos assisted tunneling occurs.

In order to study the classical-quantum correspondence we undertake a detailed study of the quantum dynamics of Eq. 3 using the technique of Floquet theory. Owing to the time-periodic nature of the system of interest, it is advantageous to obtain the Floquet states $\left|\chi_{\alpha}(t)\right\rangle=\exp \left(-i \omega_{\alpha} t\right)\left|\varphi_{\alpha}(t)\right\rangle$ and the associated quasienergies $\epsilon_{\alpha} \equiv \hbar \omega_{\alpha}$ by diagonalizing the one cycle propagator $U(T=2 \pi / \omega, 0)$. Note that the identity $U(n T, 0)=[U(T, 0)]^{n}$, due to the periodicity $\left|\varphi_{\alpha}(t+n T)\right\rangle=\left|\varphi_{\alpha}(t)\right\rangle$, implies that one can study the long time quantum dynamics using only the one cycle propagator. Moreover, the Floquet setting is ideal for interpreting the quantum dynamics in terms of the classical stroboscopic surfaces of section. In our case we obtain the quasienergies and eigenvectors by diagonalising $U(T, 0)$ in the free rotor basis $|j\rangle$.

\subsection{Control of the rotational excitations}

As mentioned earlier, a remarkable feature of the driven planar rotor system is the lack of quantum localization effects in the chaotic regions. As a result an initial rotor 


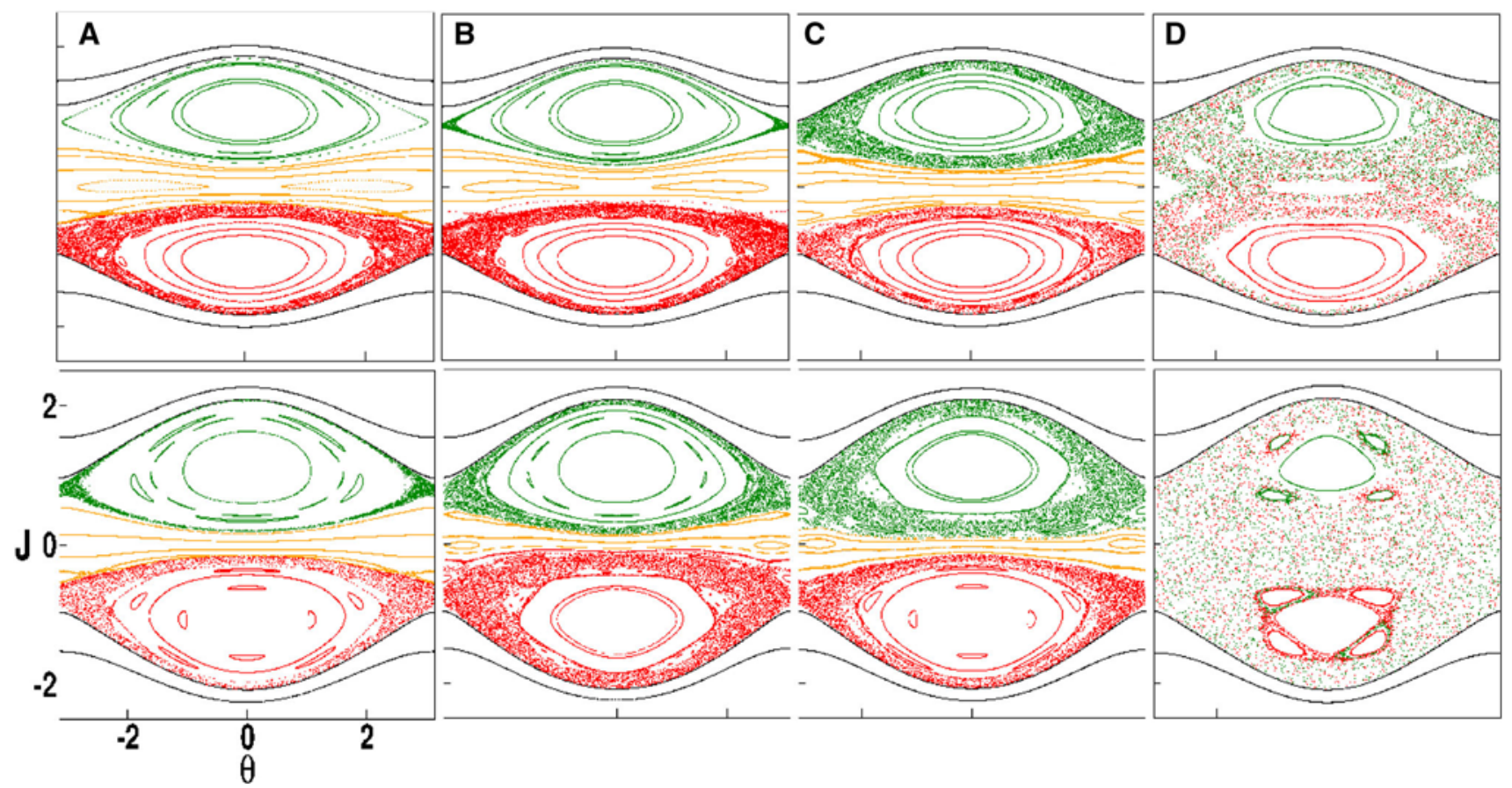

Figure 3. Stroboscopic surfaces of section showing the influence of the various Fourier modes in Eq. 13 for the control field. (A) All three modes present (B) Mode $(2,2)$ absent (C) Mode $(2,-2)$ absent (D) Mode $(2,0)$ absent. Top row corresponds to rebuilding $\omega / \Omega=2+\gamma$ barrier for $f=0.3$ while the bottom row corresponds to rebuilding the $\omega / \Omega=11+\gamma$ barrier for $f=0.5$. All the plots have identical axes range.

state $\left|j_{0}\right\rangle$ evolves under the periodic driving to yield amplitudes in very highly excited rotor levels. The maximally excited quantum state $\left|j_{\max }\right\rangle$ is solely determined by the extent of the bounded chaotic region. Clearly, introducing barriers in the chaotic sea are expected to limit the extent of rotational excitations. In our studies we have investigated the influence of the creation of barriers at various phase space locations on the classical and quantum rotational excitations for several choices of the driving field strengths, effective Planck constants, and initial rotational states. For brevity, and as representative examples, in this work we illustrate the extent to which control can be achieved with two example cases.

We choose the initial state $|j=0\rangle=\frac{1}{\sqrt{2 \pi}}$ corresponding to the ground state of the rotor with $\frac{\sqrt{2} \pi}{\hbar}=0.02$. The probability to excite the initially non-rotating diatomic molecule to the rotational state $|j\rangle$ after $n$ optical cycles i.e., $t=n T$ is given by

$$
\begin{aligned}
p_{j}(n) & =|\langle 0(n T) \mid j\rangle|^{2} \\
& =\left|\sum_{\alpha} C_{\alpha, j}^{*} C_{\alpha, 0} e^{-i n \omega_{\alpha} T}\right|^{2}
\end{aligned}
$$

where the last line is obtained by expanding the time evolution of the initial state in terms of the Floquet states and $\mathbf{C}_{\alpha}$ are the eigenvectors of the one cycle propagator. A basis of size 401 free rotor states is sufficient to obtain converged Floquet states and quasienergies for the longest timescales studied in this work.

In order to compare classical and quantum dynamics we perform the corresponding classical calculation by propagating 12000 uniformly distributed initial phase space points with $J(t=0)=0$ and $\theta \in(0,2 \pi)$. The resulting values of $J(n T)$ are then analyzed using the method used in an earlier work. ${ }^{37}$ Specifically, since $J=\hbar j$, the classical excitation probability $p_{j}^{\text {cl }}(n)$ is shown as a histogram obtained by binning the $J(n T)$ values according to $j<J(n T) / \hbar<j+1$. The bin width is taken to be equal to the effective Planck constant value.

In Figure 4A and B we compare the long time $\left(t_{f}=\right.$ $5000 T$ ) classical and quantum rotational distributions for the cases $f=0.3$ and $f=0.5$ respectively in the absence of the control field. The results can be rationalized, with the mapping $J=\hbar j$, based on Figure 1A and $\mathrm{B}$ which show the corresponding classical phase spaces. The result for $f=0.3$ shown in Figure 4A indicates that despite $\left|j_{\max }\right|$ being similar for both quantum and classical, the quantum rotational distribution is more localized as compared to the classical one. This is expected due to the regular island structures seen in Figure 1A around $J=0$. On the other hand, for the $f=0.5$ result in Figure 4B the classical and quantum distributions are similar, confirming the earlier expectation ${ }^{37,50}$ for the 


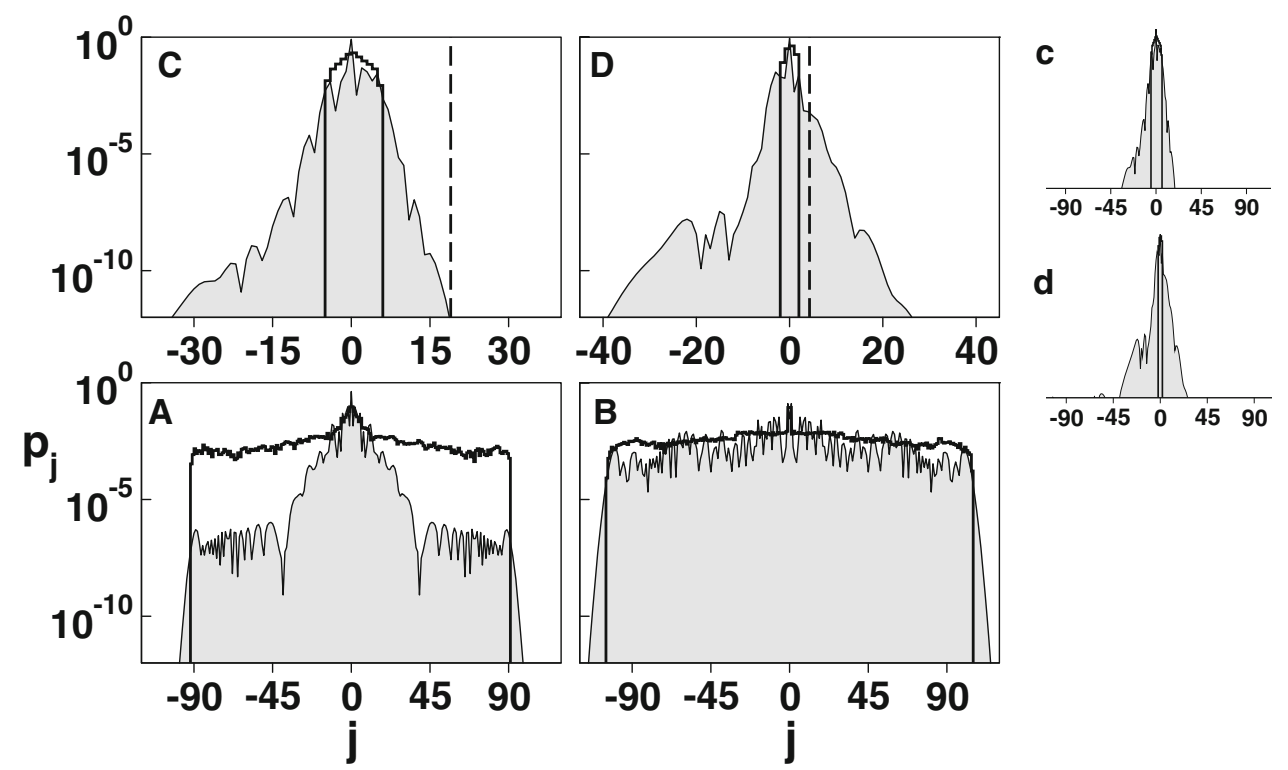

Figure 4. Quantum (shaded gray) and classical (black histogram) rotational excitation probability, at $t_{f}=5000 T$ with $\hbar=0.02$, to excite the non-rotating rotor to its $j^{\text {th }}$ rotational state. The uncontrolled cases for $f=0.3$ and $f=0.5$ are shown in (A) and (B) respectively. The corresponding results for the controlled cases are shown in (C) and (D) respectively. The phase space barriers for (C) and (D) correspond to $\omega / \Omega=2+\gamma$ and $11+\gamma$ respectively, as in Figure 3 (first column). In (C) and (D) the dashed vertical lines indicate the position of the rebuilt barriers. For comparison, the controlled results are also shown in (c) and (d) on the same scale as (A) and (B) respectively.

$p_{j}(n)$ to be nearly flat with random fluctuations. Details regarding the origins of the structures in the rotational distributions, including the short time rotational rainbow features and the long time statistical fluctuations, are already known. ${ }^{37}$ Therefore, we will not dwell upon the various features in Figure 4A and B any further and focus on the effect of the rebuilt barriers on the rotational excitations.

In Figure $4 \mathrm{C}$ and $\mathrm{D}$ we show the influence of the phase space barriers created at $J=(2+\gamma)^{-1}$ and $(11+\gamma)^{-1}$ for $f=0.3$ and 0.5 respectively. Clearly, in both cases the rotational distribution is considerably narrower as compared to the uncontrolled counterparts. One also anticipates this from the controlled phase spaces shown in Figure 3A where regular tori appear in the vicinity of the initial rotational state. In addition, the distributions are asymmetric with respect to $j=0$ which is due to the fact that the barriers are created at $J>0$. An important observation is that although both the classical and quantum excitations are controlled to a large extent, there is non-negligible quantum population in rotor levels that are not populated classically. Interestingly, while for $f=0.3$ in Figure 4C both classical and quantum populations are constrained by the barrier, for $f=0.5$ shown in Figure 4D the quantum rotor excitations do extend beyond the barrier location. Presumably this is due to dynamical tunneling through the barriers. However, this requires further considerations along the lines of the earlier studies ${ }^{55,56}$ and will be the focus of a future work.

\subsection{Control of dynamical tunneling}

The second focus of this work is to investigate the influence of the rebuilt phase space barriers on the dynamical tunneling occurring between the 1:1 resonance islands. In an earlier work, Mouchet, Eltschka, and Schlagheck have analyzed ${ }^{48}$ the various aspects of dynamical tunneling in great detail for the Hamiltonian in Eq. 3 with emphasis on the role of the nonlinear resonances. It was established that small variations of $f$ in Eq. 3 could enhance the tunneling between the two 1:1 islands by several orders of magnitude. The enhancements occur due to the emergence of nonlinear resonances within the 1:1 islands. For example, in Figure 5A and B we show the details of the phase space near the upper $1: 1$ island for $f=0.72$ and $f=0.67$ respectively. The driving field strengths considered in Figure 5 are precisely the ones that are the subject of a detailed study in the earlier work. ${ }^{48}$ It was observed that at $\hbar^{-1} \approx 16$ the tunneling for $f=0.72$ was nearly five orders of magnitude faster than that for $f=0.67$. A detailed 


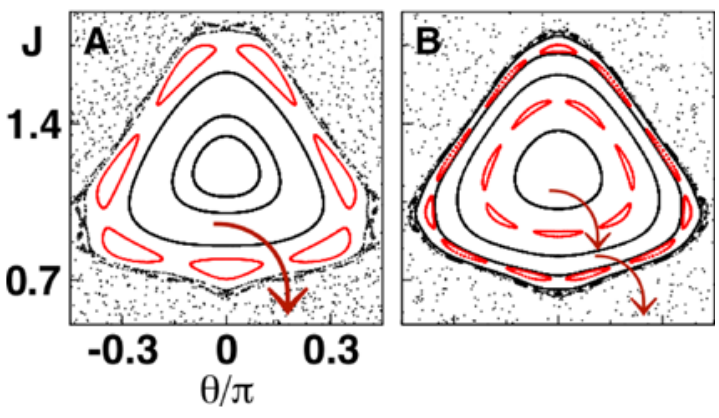

Figure 5. Details of the phase space near the 1:1 resonant island for the Hamiltonian in Eq. 3 for (A) $f=0.72$ and (B) $f=0.67$. In all the panels, curved arrows are schematic representations of resonance-assisted tunneling processes.

analysis established the cause for the enhancement to be the well developed nonlinear resonance island chain $\left(\omega / \omega_{0}=7 / 3\right.$ with $\omega_{0}$ being the frequency of oscillations around the center of the island) seen in Figure 5A. The same resonance in Figure 5B for $f=0.67$, not important at $\hbar^{-1} \approx 16$, does provide an additional pathway for resonance-assisted tunneling starting around $\hbar^{-1} \approx 30$.

Thus, dynamical tunneling in mixed regular-chaotic systems involves a combination of two processes. First, resonance-assisted tunneling ${ }^{45}$ from the 1:1 island to the chaotic sea and second, transport through the chaotic region ${ }^{47}$ due to the delocalized Floquet states. The resonance-assisted process may have several steps depending on the number of resonances that are important at a given effective $\hbar$ value. For the chaos-assisted transport part of the mechanism it is natural to surmise that the partial barriers in the chaotic sea should play an important role. In addition, recently it has been shown ${ }^{57,58}$ that delocalized Floquet states can spoil bichromatic control despite explicit symmetry breaking in the system.

Given the fact that the control term in Eq. 13 reduces the extent of the chaotic region in the phase space (cf. Figure 2 and Figure 3), one expects a drastic influence on the dynamical tunneling process. In order to confirm our expectations we take an initial state of the form

$$
\psi\left(\theta ; \theta_{c}, J_{c}\right)=\frac{1}{\left(2 \pi \sigma^{2}\right)^{1 / 4}} \exp \left[-\frac{\left(\theta_{c}-\theta\right)^{2}}{4 \sigma^{2}}+i \frac{J_{c} \theta}{\hbar}\right]
$$

localized about the upper/lower 1:1 island center $\left(\theta_{c}, J_{c}\right)$ with $\sigma^{2}=\hbar / 2$. Note that the above state is similar to an appropriately squeezed coherent state and we are working with dimensionless units. A signature of resonance and chaos assisted tunneling is the interaction of the tunneling doublet with one or more states,
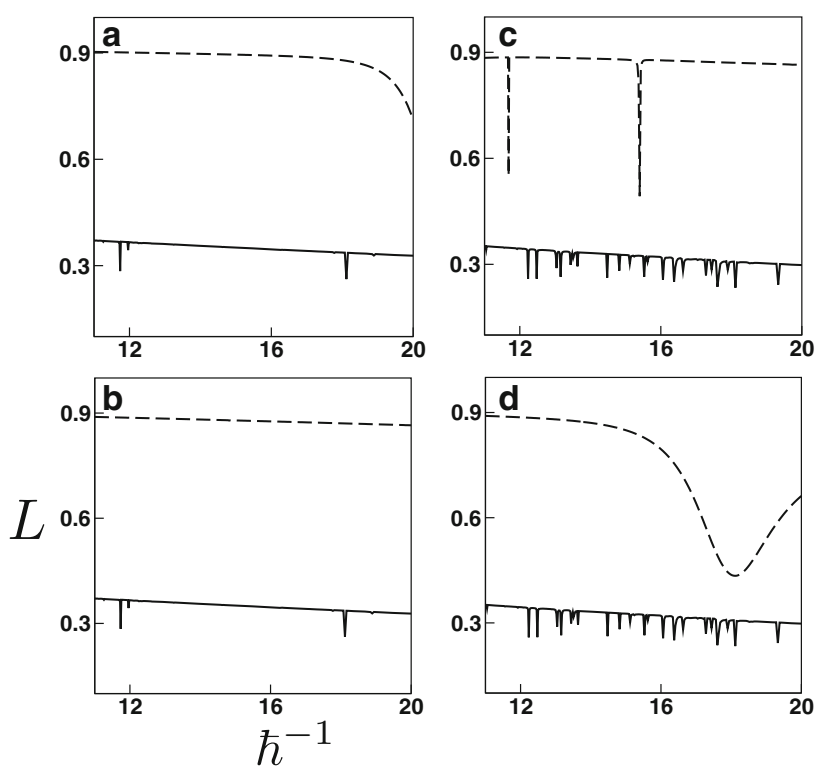

Figure 6. Long time limit of the survival probability Eq. 19 for the uncontrolled (solid line) and controlled (dashed line) cases. The initial state is localized in the lower $\left(I_{-}\right)$and upper ( $I_{+}$) 1:1 island in the classical phase space. (a) $f=0.67, I_{+}$ (b) $f=0.67, I_{-}$(c) $f=0.72, I_{+}$(d) $f=0.72, I_{-}$. The rebuilt barrier corresponds to $\omega / \Omega=11+\gamma$ in all cases and $\hbar=0.02$.

regular or chaotic, leading to fluctuations in the tunnel splittings. A convenient measure for the number of states that participate in the dynamics of an initial state is the long time limit of the survival probability

$$
\begin{aligned}
L & \equiv \lim _{\tau \rightarrow \infty}|\langle\psi(0) \mid \psi(\tau)\rangle|^{2} \\
& =\lim _{\tau \rightarrow \infty} \sum_{\alpha, \beta} p_{\alpha} p_{\beta} e^{-i\left(\epsilon_{\alpha}-\epsilon_{\beta}\right) \tau / \hbar}=\sum_{\alpha} p_{\alpha}^{2}
\end{aligned}
$$

with $p_{\alpha} \equiv\left|\left\langle\psi(0) \mid \varphi_{\alpha}(0)\right\rangle\right|^{2}$ being the overlap intensity. The participation ratio $L^{-1}$ represents the number of Floquet states that participate in the dynamics of $|\psi(0)\rangle$. In a typical two level dynamics involving only the tunneling doublet $L \approx 0.5$ whereas for the participation of additional states one expects $L<0.5$. Moreover, $L \approx 1$ indicates the lack of any tunneling and the initial state is localized. Recently $L$ has been used ${ }^{57}$ to map the tunneling control landscape as a function of the various driving field parameters. In order to show the usefulness of Eq. 19, Figure 6 shows $L$ as a function of $\hbar^{-1}$ for our system in the absence of any control term. One can clearly see in Figure 6(d), corresponding to $f=0.72$, a large number of multistate interactions with $L \lesssim 0.3$. In contrast, the uncontrolled result for $f=0.67$ in Figure 6(a) shows similar $L$ values but with isolated multistate interactions. These 

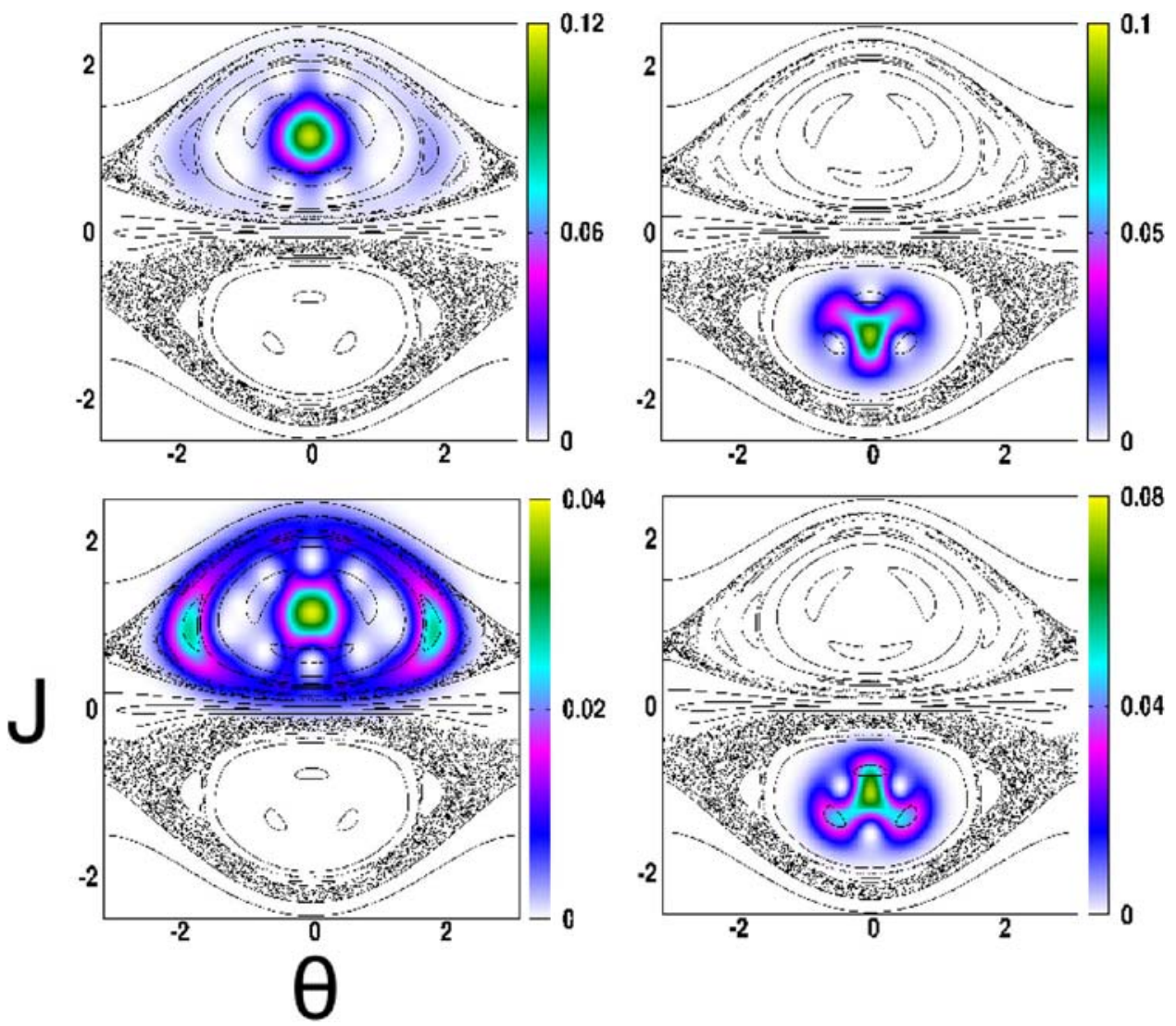

Figure 7. Husimi distributions Eq. 20 of select states that lead to the loss of control seen in Figure 6 for $f=0.72$ and $\hbar=0.02$. First column shows the states responsible for the sharp dip in $L$ around $\hbar^{-1} \approx 15$ in Figure 6(c) and the second column shows the states responsible for the broad dip in $L$ around $\hbar^{-1} \approx 18$ in Figure $6(\mathrm{~d})$. Note that the Husimi distributions are superimposed on the controlled phase space for comparison.

results are consistent with the earlier observations ${ }^{48}$ where the orders of magnitude enhancement of tunneling for $f=0.72$ as compared to $f=0.67$ was ascribed to the multitude of crossings involving delocalized Floquet states.

In Figure 6 we show the effect of rebuilding the $\omega / \Omega=11+\gamma$ barrier on dynamical tunneling for the coupling strengths $f=0.67$ and 0.72 with varying $\hbar^{-1}$ values. The chosen barrier is nearly symmetric with respect to the $1: 1$ islands and the specific choice of the coupling parameters is made to make contact with the earlier work. In Figure 6(a) and (b) we show the result for $f=0.67$ with the initial state localized in the upper and lower islands respectively. Clearly, tunneling is controlled quite well over the range of $\hbar$ values considered. In contrast, similar computations for $f=0.72$ shown in Figure 6(c) and (d) indicate a few isolated regions where the control is not effective. It is important to note that the multistate interactions are not observed with the control term being present. This is consistent with the fact that the rebuilt barrier has significantly constrained the chaotic regions in the phase space (cf. Figure 7 for the controlled phase space).

In order to understand the origins of the lack of control seen in Figure 6(c) and (d) we compute the Husimi distribution functions for the Floquet states of interest and compare to the classical phase space structures. The Husimi distribution ${ }^{59}$

$$
\rho_{H}(\theta, J)=\frac{1}{2 \pi}\left|\left\langle\chi_{\alpha} \mid G\right\rangle\right|^{2},
$$

is given by the overlap of coherent states $|G\rangle$ (similar to Eq. 18) localized at various phase space points $(\theta, J)$ with the Floquet states $\left|\chi_{\alpha}\right\rangle$. In Figure 7 first column we show the Husimi distribution of the two states that have the largest overlap with the initial state localized in the upper 1:1 island. These two states correspond to the dip in $L$ seen in Figure 6(c) at $\hbar^{-1} \approx 15$. One can clearly see that a Floquet state localized on one of the resonances within the 1:1 island interacts with the initial state leading to the loss of control. Nevertheless, it is important to see that the rebuilt torus is acting as a barrier since there is no Husimi density in the 1:1 island below. Similarly, 
Figure 7 second column shows that a different island chain within the main 1:1 resonance is is leading to the loss of control observed in Figure 6(d) as a broad dip for $\hbar^{-1} \approx 18$. Notably, in both instances the dynamical tunneling is indeed controlled by the rebuilt barrier. However, isolated interactions with certain regular Floquet states within the 1:1 island lead to the "leaking" of the initial state.

\section{Conclusions}

In this work we have shown, building on our earlier work, ${ }^{36}$ that one can control both the classical and the corresponding quantum dynamics by rebuilding barriers in the classical phase space. Specifically, using the example of a driven planar rotor, it is possible to control both the rotational excitations and dynamical tunneling in the system. Note that the control of dynamical tunneling in this context amounts to localizing a diatomic molecule in regions of phase space with limited angular excursions about, and aligned with or against, the field axis. Such "pendular" states have been the subject of several studies. Indeed, one route to molecular alignment involves the use of a strong dc-field ${ }^{60,61}$ and it is remarkable that our classical control term Eq. 13 has such a component. Moreover, as evident from Figure 3, the dc-field component in the control Hamiltonian is crucial and the coefficient of the $(2,0)$ Fourier mode in Eq. 13 can be related to the polarizability anisotropy of the diatomic molecule. Thus, one anticipates a close classical-quantum correspondence for the interplay ${ }^{62}$ of rotational excitations and molecular alignment.

The current study is concerned with building a single phase space barrier. Nevertheless, it is possible to construct several barriers together at appropriate locations using a similar approach. It would be interesting to explore the consequences of such multiple barriers on the rotational dynamics. The values of the effective Planck constant used here implies that the results are relevant, as noted before, ${ }^{50}$ to rather heavy diatomic molecules like cesium iodide (CsI). It remains to be seen whether our approach can be suitably modified to be applicable to lighter diatomics like carbon monoxide (CO). Moreover, the generalization of the perturbative approach to pulsed and tilted fields ${ }^{63}$ is worth exploring since the role ${ }^{26,64}$ of the sub Planck scale classical phase space structures may be different when compared to a system driven by an ac-field.

\section{Acknowledgements}

AS thanks the Council for Scientific and Industrial Research (CSIR), India for a doctoral fellowship.

\section{References}

1. Tannor D J and Rice S A 1985 Control of selectivity of chemical reaction via control of wavepacket evolution $J$. Chem. Phys. 835013

2. Shapiro M, Hepburn J W and Brumer P 1988 Simplified laser control of unimolecular reactions: simultaneous $\left(\omega_{1}, \omega_{3}\right)$ excitation Chem. Phys. Lett. 149451

3. Judson R S and Rabitz H 1992 Teaching lasers to control molecules Phys. Rev. Lett. 681500

4. Daniel C et al. 2003 Deciphering the reaction dynamics underlying optimal control laser fields Science 299536

5. Langhojer F, Cardoza D, Baertschy M and Weinacht T 2005 Gaining mechanistic insight from closed loop learning control: the importance of basis in searching the space J. Chem. Phys. 122014102

6. Wollenhaupt M, Präkelt A, Sarpe-Tudoran C, Liese D and Baumert T 2005 Quantum control and quantum control landscapes using intense shaped femtosecond pulses J. Mod. Opt. 522187

7. Marquetand P and Engel V 2008 Analysis of laser fields for photoassociation and molecular stabilization derived from local control theory J. Phys. B: At. Mol. Opt. Phys. 41074026

8. Rice S A 2001 Interfering for the good of a chemical reaction Nature 409422

9. Franco I, Spanner M and Brumer P 2010 quantum interferences and their classical limit in laser driven coherent control scenarios Chem. Phys. 370143

10. Franco I and Brumer P 2006 Laser-induced spatial symmetry breaking in quantum and classical mechanics Phys. Rev. Lett. 97040402

11. Flach S, Yevtushenko O and Zolotrayuk Y 2000 Directed current due to broken time-space symmetry Phys. Rev. Lett. 842358

12. Ivanov M, Bartram D and Smirnova O 2012 Coherent control in strongly driven multi-level systems: quantum vs classical features Mol. Phys. 1101801

13. Franco I and Brumer P 2008 Minimum requirements for laser-induced symmetry breaking in quantum and classical mechanics J. Phys. B: At. Mol. Opt. Phys. 41 074003

14. van Leeuwen $\mathrm{K}$ A H et al. 1985 Microwave ionization of hydrogen atoms: experiment versus classical dynamics Phys. Rev. Lett. 552231

15. Brown R C and Wyatt R E 1985 Quantum mechanical manifestation of cantori: wave-packet localization in stochastic regions Phys. Rev. Lett. 571

16. Liu W-K, Wu B and Yuan J-M 1995 Nonlinear dynamics of chirped pulse excitation and dissociation of diatomic molecules Phys. Rev. Lett. 751292

17. Davis M J and Heller E J 1981 Quantum Dynamical Tunneling in Bound States J. Chem. Phys. 75246

18. Lakshminarayan A 2001 Entangling power of quantized chaotic systems Phys. Rev. E 64036207

19. Casati G, Chirikov B V, Shepelyansky D L and Guarneri I 1987 Relevance of classical chaos in quantum mechanics: the hydrogen atom in a monochromatic field Phys. Rep. 15479

20. Bohigas O, Tomsovic S and Ullmo D 1993 Manifestations of classical phase space structures in quantum mechanics Phys. Rep. 22343 
21. Holthaus M 1995 On the classical-quantum correspondence for periodically time dependent systems Chaos, Solitons \& Fractals 51143

22. Grifoni M and Hänggi P 1998 Driven quantum tunneling Phys. Rep. 304229

23. Buchleitner A, Delande D and Zakrzewski J 2002 Nondispersive wave packets in periodically driven quantum systems Phys. Rep. 368409

24. Brodier O, Schlagheck P and Ullmo D 2002 Resonanceassisted tunneling Ann. Phys. 30088

25. Bird J P et al. 2003 Interference and interactions in open quantum dots Rep. Prog. Phys. 66583

26. Gong J and Brumer P 2005 Quantum chaos meets coherent control Annu. Rev. Phys. Chem. 561

27. Keshavamurthy S 2007 Dynamical Tunneling in Mole cules: Quantum Routes to Energy Flow Int. Rev. Phys. Chem. 26521

28. Jacquod Ph and Petitjean C 2009 Decoherence, entanglement and irreversibility in quantum dynamical systems with few degrees of freedom Adv. Phys. 5867

29. Denisov S, Flach S and Hänggi P 2014 Tunable transport with broken space-time symmetries Phys. Rep. 538 77

30. Ciraolo $\mathrm{G}$ et al. 2004 Controlling chaotic transport in a Hamiltonian model of interest to magnetized plasmas $J$. Phys. A: Math. Gen. 373589

31. Chandre C et al. 2005 Channeling chaos by building barriers Phys. Rev. Lett. 94074101

32. Vittot M, Chandre C, Ciraolo G and Lima R 2005 Localized control for non-resonant Hamiltonian systems Nonlinearity 18423

33. Huang S, Chandre C and Uzer T 2006 Reducing multiphoton ionization in a linearly polarized microwave field by local control Phys. Rev. A 74053408

34. Zion B Y and Horwitz L 2010 Controlling effect of geometrically defined local structural changes on chaotic Hamiltonian systems Phys. Rev. E 81046217

35. de Sousa M C, Caldas I L, Rizzato F B, Pakter R and Steffens F M 2012 Controlling chaos in wave-particle interactions Phys. Rev. E 86016217

36. Sethi A and Keshavamurthy S 2009 Local phase space control and interplay of classical and quantum effects in dissociation of a driven Morse oscillator Phys. Rev. A 79 033416

37. Moiseyev N, Korsch H J and Mirbach B 1994 Classical and quantum chaos in molecular rotational excitation by ac electric fields Z. Phys. D 29125

38. Mouchet A, Miniatura C, Kaiser R, Grémaud B and Delande D 2001 Chaos assisted tunneling with cold atoms Phys. Rev. E 64016221

39. Hensinger W K et al. 2001 Dynamical tunneling of cold atoms Nature $\mathbf{4 1 2} 52$

40. Steck D A, Oskay W H and Raizen M G 2002 Observations of chaos-assisted tunneling between islands of stability Science 293274

41. Chirikov B V 1979 A universal instability of many-dimensional oscillator systems Phys. Rep. 52 263

42. Berman G P and Kolovsky A R 1983 Structure and stability of the quasi-energy spectrum of two interacting quantum nonlinear resonances Phys. Lett. 95A 15
43. Berman G P, Zaslavsky G M and Kolovsky A R 1982 On the spectrum of the system of interacting quantum nonlinear resonances Phys. Lett. 87A 152

44. Kolovsky A R 1991 Regular and chaotic dynamics of a molecule affected by an external resonance field Phys. Lett. A 157474

45. Brodier O, Schlagheck P and Ullmo D 2001 ResonanceAssisted Tunneling in Near-Integrable Systems Phys. Rev. Lett. 87064101

46. Keshavamurthy S 2005 On dynamical tunneling and classical resonances J. Chem. Phys. 122114109

47. Tomsovic S and Ullmo D 1994 Chaos Assisted Tunneling Phys. Rev. E 50145

48. Mouchet A, Eltschka C and Schlagheck P 2006 Influence of classical resonances on chaotic tunneling Phys. Rev. E 74026211

49. Fishman S, Grempel D R and Prange R E 1982 Chaos, quantum recurrences, and Anderson localization Phys. Rev. Lett. 49509

50. B1ümel R, Fishman S and Smilansky U 1985 Excitation of molecular rotation by periodic microwave pulses. A testing ground for Anderson localization J. Chem. Phys. 842604

51. Casati G, Chirikov B V and Shepelyansky D L 1984 Quantum Limitations for Chaotic Excitation of the Hydrogen Atom in a Monochromatic Field Phys. Rev. Lett. $\mathbf{5 3} 2525$

52. Brivio G P, Casati G, Perotti L and Guarneri I 1988 Quantum suppression of chaotic diffusion: Theory and experiment Physica D $\mathbf{3 3} 51$

53. Escande D F and Doveil F 1981 Renormalization method for computing the threshold of the large-scale stochastic instability in two degrees of freedom Hamiltonian systems J. Stat. Phys. 26257

54. MacKay R S and Meiss J D 1988 Relation between quantum and classical thresholds for multiphoton ionization of excited atoms Phys. Rev. A 374702

55. Geisel T, Radons G and Rubner J 1986 KolmogorovArnol'd-Moser barriers in the quantum dynamics of chaotic systems Phys. Rev. Lett. 572883

56. Maitra N T and Heller E J 2000 Quantum transport through cantori Phys. Rev. E 613620

57. Shukla A and Keshavamurthy S 2015 One versus two photon control of dynamical tunneling: Influence of the irregular Floquet states J. Phys. Chem. B 11911326

58. Sethi A and Keshavamurthy S 2008 Bichromatically driven double well: Parametric perspective of the strong field control landscape reveals the influence of chaotic states J. Chem. Phys. 128164117

59. Takahashi K and Saito N 1985 Chaos and Husimi distribution in quantum mechanics Phys. Rev. Lett. 55 645

60. Friedrich B and Herschbach D 1999 Manipulating molecules via combined static and laser fields J. Phys. Chem. A 10310280

61. Friedrich B and Herschbach D 1999 Enhanced orientation of polar molecules by combined electrostatic and nonresonant induced dipole forces J. Chem. Phys. 111 6157

62. Seideman T 1995 Rotational excitation and molecular alignment in intense laser fields J. Chem. Phys. 103 7887 
63. Arango C A, Kennerly W W and Ezra G S 2005 Classical and quantum mechanics of diatomic molecules in tilted fields J. Chem. Phys. 122 184303
64. Perotti L C 2010 Small phase-space structures and their relevance to pulsed quantum evolution: stepwise ionization of the excited hydrogen atom in a microwave pulse Phys. Rev. A 81033407 